\title{
BEHAVIOR OF CORANK-ONE SINGULAR POINTS ON WAVE FRONTS
}

\author{
Kentaro SAJI, Masaaki UMEHARA and Kotaro YAMADA
}

(Received 20 April 2007)

\begin{abstract}
Let $M^{2}$ be an oriented 2-manifold and $f: M^{2} \rightarrow \mathbb{R}^{3}$ a $C^{\infty}$-map. A point $p \in M^{2}$ is called a singular point if $f$ is not an immersion at $p$. The map $f$ is called a front (or wave front), if there exists a unit $C^{\infty}$-vector field $v$ such that the image of each tangent vector $d f(X)\left(X \in T M^{2}\right)$ is perpendicular to $v$, and the pair $(f, v)$ gives an immersion into $\mathbb{R}^{3} \times S^{2}$. In a previous paper, we gave an intrinsic formulation of wave fronts in $\mathbb{R}^{3}$. In this paper, we investigate the behavior of cuspidal edges near corank-one singular points and establish Gauss-Bonnet-type formulas under the intrinsic formulation.
\end{abstract}

\section{Introduction}

Let $M^{2}$ be a 2-manifold and $f: M^{2} \rightarrow \mathbb{R}^{3}$ a $C^{\infty}$-map. A point $p \in M^{2}$ is called regular if $f$ is an immersion on a sufficiently small neighborhood of $p$, and is called singular if it is not regular. To extend the concept of surfaces to a larger class that allows singularities, we recall the following definitions. A $C^{\infty}$-map $f: M^{2} \rightarrow \mathbb{R}^{3}$ is called a frontal if there exists a unit vector field $v$ along $f$ such that $v$ is perpendicular to $d f\left(T M^{2}\right)$. By parallel translations, $v$ can be considered as a map into the unit sphere $S^{2}$, which is called the Gauss map of the frontal $f$. Moreover, if the map

$$
L:=(f, v): M^{2} \longrightarrow \mathbb{R}^{3} \times S^{2}
$$

gives an immersion, $f$ is called a front or a wave front. Using the canonical inner product on $\mathbb{R}^{3}$, we identify the unit tangent bundle $\mathbb{R}^{3} \times S^{2}=T_{1} \mathbb{R}^{3}$ with the unit cotangent bundle $T_{1}^{*} \mathbb{R}^{3}$, which has the canonical contact structure. When $f$ is a front, $L$ gives a Legendrian immersion with respect to the canonical contact structure. Hence, fronts are considered as projections of Legendrian immersions.

Consider a front $f: M^{2} \rightarrow \mathbb{R}^{3}$ defined on a compact oriented 2-manifold $M^{2}$. When the set $\Sigma$ of singular points of $f$ consists of cuspidal edges and swallowtails, Langevin-LevittRosenberg [LLR] and Kossowski [K] proved the following two Gauss-Bonnet-type formulas

$$
\begin{gathered}
2 \operatorname{deg}(v)=\chi\left(M_{+}\right)-\chi\left(M_{-}\right)+\# S_{+}-\# S_{-} \quad(\text { see }[\mathbf{L L R}, \mathbf{K}]), \\
2 \pi \chi\left(M^{2}\right)=\int_{M^{2}} K d A+2 \int_{\Sigma} \kappa_{s} d \tau \quad(\text { see }[\mathbf{K}]),
\end{gathered}
$$

2000 Mathematics Subject Classification: Primary 57R45;

$$
\text { Secondary 53A05. }
$$

Keywords and Phrases: wave fronts; singular curvature; coherent tangent bundles; Gauss-Bonnet theorem. 
where $\operatorname{deg}(v)$ is the degree of the Gauss map, $\# S_{+}, \# S_{-}$are the numbers of positive and negative swallowtails, respectively, $M_{+}$(respectively $M_{-}$) is the open submanifold of $M^{2}$ to which the co-orientation is compatible (respectively not compatible) with respect to the orientation, and $d A$ (respectively $d \tau$ ) is the area element of the surface (respectively the arclength measure of the singular set). (See Section 1 or [SUY] for precise definitions.) The function $\kappa_{s}$ is called the singular curvature function, which was originally defined in [SUY]. In the proofs of these formulas in [LLR] and $[\mathbf{K}]$, the singular curvature implicitly appeared as a measure $\kappa_{s} d \tau$. (The formula (1) is stated in [LLR], and proofs for both (1) and (2) are in $[\mathbf{K}]$.)

In [SUY], the authors stated a generalization of (1) and (2) for singularities containing double swallowtails, and gave a sketch of their proofs.

On the other hand, the classical Gauss-Bonnet formula is intrinsic in nature. So it is quite natural to formulate the singularities of fronts intrinsically. In this paper, we give a general setting of intrinsic fronts according to the final section of [SUY], and prove the intrinsic Gauss-Bonnet formulas (Theorem B in Section 2). As a consequence, our intrinsic approach also gives a detailed explanation of the proofs of (2.2), (2.6) and Theorem 2.3 in [SUY] (see Theorem A and Theorem B in Section 2).

\section{An intrinsic approach and the singular curvature function}

In this section, we give a general setting for intrinsic wave fronts.

Definition 1.1. Let $M^{2}$ be an oriented 2-manifold. An orientable vector bundle $\mathcal{E}$ of rank 2 with a metric $\langle\cdot, \cdot\rangle$ and a metric connection $D$ is called an abstract limiting tangent bundle or a coherent tangent bundle if there is a bundle homomorphism

$$
\psi: T M^{2} \longrightarrow \mathcal{E}
$$

such that

$$
D_{X} \psi(Y)-D_{Y} \psi(X)=\psi([X, Y]) \quad\left(X, Y \in T M^{2}\right) .
$$

In [SUY], the authors used the term an abstract limiting tangent bundle, but in this paper we use coherent tangent bundle instead, since it is a shorter phrase.

In this setting, the pull-back of the metric $d s^{2}:=\psi^{*}\langle\cdot, \cdot\rangle$ is called the first fundamental form of $\psi$. A point $p \in M^{2}$ is called a singular point (of $\psi$ ) if $\psi_{p}: T_{p} M^{2} \rightarrow \mathcal{E}_{p}$ is not a bijection, where $\mathcal{E}_{p}$ is the fiber of $\mathcal{E}$ at $p$, that is, the first fundamental form is not positive definite. We denote by $\Sigma$ the set of singular points on $M^{2}$.

Since $\mathcal{E}$ is orientable, there exists a smooth non-vanishing skew-symmetric bilinear section $\mu \in \operatorname{Sec}\left(\mathcal{E}^{*} \wedge \mathcal{E}^{*}\right)$ such that $\mu\left(e_{1}, e_{2}\right)= \pm 1$ for any orthonormal frame $\left\{e_{1}, e_{2}\right\}$ on $\mathcal{E}$. The form $\mu$ is determined uniquely up to \pm -ambiguity. A co-orientation of the coherent tangent bundle $\mathcal{E}$ is a choice of $\mu$. A frame $\left\{e_{1}, e_{2}\right\}$ is called positive with respect to the co-orientation $\mu$ if $\mu\left(e_{1}, e_{2}\right)=+1$.

From now on, we fix a co-orientation $\mu$ on the coherent tangent bundle.

Definition 1.2. (Area elements) The signed area form $d \hat{A}$ and the (unsigned) area form $d A$ are defined on a positively oriented local coordinate system $(U ; u, v)$ on $M^{2}$ as

$$
d \hat{A}:=\psi^{*} \mu=\lambda d u \wedge d v, \quad d A:=|\lambda| d u \wedge d v,
$$


where

$$
\lambda:=\mu\left(\psi_{u}, \psi_{v}\right), \quad\left(\psi_{u}:=\psi\left(\frac{\partial}{\partial u}\right), \psi_{v}:=\psi\left(\frac{\partial}{\partial v}\right)\right) .
$$

We call the function $\lambda$ the signed area density function on $U$. The set of the singular points on $U$ is expressed as

$$
\Sigma \cap U:=\{p \in U ; \lambda(p)=0\} .
$$

Both $d \hat{A}$ and $d A$ are independent of the choice of positively oriented local coordinate system $(u, v)$, and give globally defined 2-forms on $M^{2}\left(d \hat{A}\right.$ is $C^{\infty}$-differentiable, but $d A$ is only continuous). When $M^{2}$ has no singular points, the two forms coincide up to sign. We set

$$
M_{+}:=\left\{p \in M^{2} \backslash \Sigma ; d \hat{A}_{p}=d A_{p}\right\}, \quad M_{-}:=\left\{p \in M^{2} \backslash \Sigma ; d \hat{A}_{p}=-d A_{p}\right\} .
$$

The singular set $\Sigma$ coincides with $\partial M_{+}=\partial M_{-}$.

A singular point $p \in \Sigma$ is called non-degenerate if $d \lambda$ does not vanish at $p$. On a neighborhood of a non-degenerate singular point, the singular set consists of a regular curve $\gamma(t)$ on $M^{2}$, called the singular curve. The tangential direction of the singular curve is called the singular direction. If $p$ is a non-degenerate singular point, the rank of $\psi_{p}$ is 1 . The direction of the kernel of $\psi_{p}$ is called the null direction. Let $\eta(t)$ be the smooth (nonvanishing) vector field along the singular curve $\gamma(t)$ which gives the null direction.

Here, we give an example.

Example 1.3. Let $M^{2}$ be an oriented 2-manifold, $f: M^{2} \rightarrow \mathbb{R}^{3}$ a frontal (see the introduction) and $v: M^{2} \rightarrow S^{2}$ its unit normal vector field. Then the vector bundle $\mathcal{E}^{f}$ on $M^{2}$ whose fiber at $p \in M^{2}$ is given by

$$
\mathcal{E}_{p}^{f}:=\left\{X \in T_{f(p)} \mathbb{R}^{3} ; X \text { is perpendicular to } v(p)\right\}
$$

is called the limiting tangent bundle of $f$. The restriction of the canonical inner product on $\mathbb{R}^{3}$ gives the metric on $\mathcal{E}^{f}$, and the tangential part of the Levi-Civita connection on $\mathbb{R}^{3}$ gives the covariant derivative on $\mathcal{E}^{f}$ satisfying the condition (1.1). The bundle homomorphism between $T M^{2}$ and $\mathcal{E}^{f}$ is given by

$$
\psi_{p}(X):=d f_{p}(X) \in \mathcal{E}_{p}^{f} \quad\left(X \in T_{p} M^{2}, p \in M^{2}\right) .
$$

We call this $\mathcal{E}^{f}$ the coherent tangent bundle associated with the frontal $f$. Let $(U ; u, v)$ be an arbitrary positively oriented local coordinate system of $M^{2}$. Then the determinant of three vectors

$$
\lambda:=\operatorname{det}\left(f_{u}, f_{v}, v\right) \quad\left(f_{u}:=\frac{\partial f}{\partial u}, f_{v}:=\frac{\partial f}{\partial v}\right)
$$

gives the signed area density function on $U$.

A singular point is called a cuspidal edge or a swallowtail if the corresponding germ of the $C^{\infty}$-map is $\mathcal{A}$-equivalent to that of the $C^{\infty}$-map germ

$$
f_{\mathrm{C}}(u, v):=\left(u^{2}, u^{3}, v\right) \quad \text { or } \quad f_{\mathrm{SW}}(u, v):=\left(3 u^{4}+u^{2} v, 4 u^{3}+2 u v, v\right)
$$

at $(u, v)=(0,0)$, respectively (see Figure 1). Here, two $C^{\infty}$-maps $f_{i}: U_{i} \rightarrow \mathbb{R}^{3}(i=1,2)$ are $\mathcal{A}$-equivalent (or right-left equivalent) at the points $p_{i} \in U_{i} \subset \mathbb{R}^{2}(i=1,2)$ if there exists a local diffeomorphism $\varphi$ of $\mathbb{R}^{2}$ with $\varphi\left(p_{1}\right)=p_{2}$ and a local diffeomorphism $\Phi$ of $\mathbb{R}^{3}$ with 

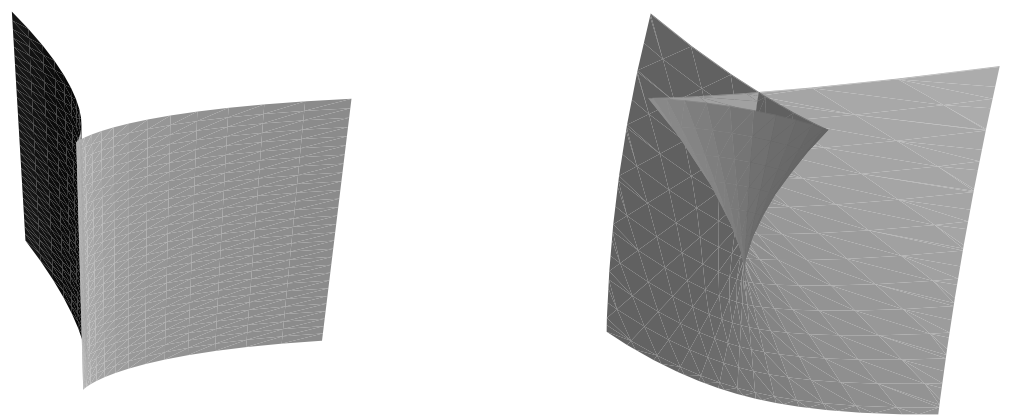

FIGURE 1. A cuspidal edge and a swallowtail.

$\Phi\left(f_{1}\left(p_{1}\right)\right)=f_{2}\left(p_{2}\right)$ such that $f_{2}=\Phi \circ f_{1} \circ \varphi^{-1}$. It can be easily checked that both $f_{\mathrm{C}}$ and $f_{\mathrm{SW}}$ are fronts. These two types of singular points characterize the generic singularities of fronts in $\mathbb{R}^{3}$. The singular curve of $f_{\mathrm{C}}$ is the $v$-axis and the null direction is the $u$ direction. The singular curve of $f_{\mathrm{SW}}$ is the parabola $6 u^{2}+v=0$ and the null direction is the $u$-direction.

Definition 1.4. Let $(\mathcal{E},\langle\cdot, \cdot\rangle, D, \psi)$ be a coherent tangent bundle as in Definition 1.1. Take a non-degenerate singular point $p \in M^{2}$ and let $\gamma(t)$ be the singular curve satisfying $\gamma(0)=p$. Then $p$ is called an $A_{2}$-point or an intrinsic cuspidal edge if the null direction $\eta(0)$ is transversal to the singular direction $\dot{\gamma}(0)=d \gamma /\left.d t\right|_{t=0}$. If $p$ is not an $A_{2}$-point, but satisfies

$$
\left.\frac{d}{d t}\right|_{t=0}(\dot{\gamma}(t) \wedge \eta(t)) \neq 0,
$$

it is called an $A_{3}$-point or an intrinsic swallowtail, where $\wedge$ is the exterior product on $T M^{2}$.

FACT 1.5. (Kokubu et al [KRSUY]) Suppose that $f: M^{2} \rightarrow \mathbb{R}^{3}$ is a front. Then a nondegenerate singular point $p \in M^{2}$ is a cuspidal edge (respectively swallowtail) if and only if it is an $A_{2}$-point (respectively an $A_{3}$-point).

Remark 1.6. A cuspidal cross cap is a singular point which is $\mathcal{A}$-equivalent to the $C^{\infty}$-map germ

$$
f_{\mathrm{CCR}}(u, v):=\left(u, v^{2}, u v^{3}\right),
$$

at $(u, v)=(0,0)$, see Figure 2 . The map $f_{\mathrm{CCR}}$ is not a front but a frontal with the unit normal vector field

$$
v_{\mathrm{CCR}}:=\frac{1}{\sqrt{4+9 u^{2} v^{2}+4 v^{6}}}\left(-2 v^{3},-3 u v, 2\right) .
$$

Although a cuspidal cross cap is different from cuspidal edge, it is also an $A_{2}$-point in the sense of Definition 1.4. So Fact 1.5 requires the assumption that $f$ is a front. (A useful criterion for cuspidal cross caps is given in [FSUY].)

Now we take a coherent tangent bundle $(\mathcal{E},\langle\cdot, \cdot\rangle, D, \psi)$ and fix a singular curve $\gamma(t)$ consisting of $A_{2}$-points. Since $d \gamma / d t$ is transversal to the null direction, the image $\psi(d \gamma / d t)$ 


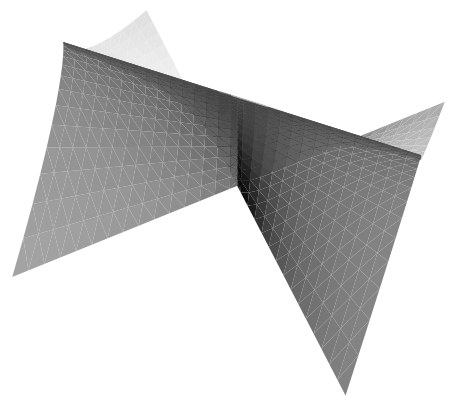

FIGURE 2. A cuspidal crosscap.

does not vanish, and then we can take a parameter $\tau$ of $\gamma$ such that

$$
\left\langle\psi\left(\gamma^{\prime}(\tau)\right), \psi\left(\gamma^{\prime}(\tau)\right)\right\rangle=1 \quad\left({ }^{\prime}=\frac{d}{d \tau}\right),
$$

which is called the arclength parameter of the singular curve $\gamma$. Take a null vector field $\eta(\tau)$ along $\gamma(\tau)$ such that $\left\{\gamma^{\prime}(\tau), \eta(\tau)\right\}$ is a positively oriented frame field along $\gamma$ for each $\tau$.

Let $n(\tau)$ be a section of $\mathcal{E}$ along $\gamma(\tau)$ such that $\left\{\psi\left(\gamma^{\prime}(\tau)\right), n(\tau)\right\}$ is a positive orthonormal frame, which is called the $\mathcal{E}$-conormal of $\gamma$. Then

$$
\hat{\kappa}_{g}(\tau):=\left\langle D_{\tau} \psi\left(\gamma^{\prime}(\tau)\right), n(\tau)\right\rangle=\mu\left(\psi\left(\gamma^{\prime}(\tau)\right), D_{\tau} \psi\left(\gamma^{\prime}(\tau)\right)\right)
$$

is called the $\mathcal{E}$-geodesic curvature of $\gamma$, which gives the geodesic curvature of the singular curve $\gamma$ with respect to the orientation of $\mathcal{E}$, where $D_{\tau}=D_{d / d \tau}$. Then the singular curvature function is defined by

$$
\kappa_{S}(\tau):=\operatorname{sgn}(d \lambda(\eta(\tau))) \hat{\kappa}_{g}(\tau)
$$

where $\operatorname{sgn}(d \lambda(\eta(\tau)))$ denotes the sign of the function $d \lambda(\eta)$ at $\tau$. In a general parameterization of $\gamma=\gamma(t)$, the singular curvature function is

$$
\kappa_{S}(t)=\operatorname{sgn}(d \lambda(\eta(t))) \frac{\mu\left(\psi(\dot{\gamma}(t)), D_{t} \psi(\dot{\gamma}(t))\right)}{|\psi(\dot{\gamma}(t))|^{3}} \quad\left(\cdot=\frac{d}{d t}\right),
$$

where $|\xi|:=\sqrt{\langle\xi, \xi\rangle}$ denotes the norm derived from the metric $\langle\cdot, \cdot\rangle$.

PROPOSITION 1.7. (An intrinsic version of [SUY, Theorem 1.6]) The singular curvature function does not depend on the orientation of $M^{2}$, the orientation of $\mathcal{E}$ nor the parameter $t$ of the singular curve $\gamma(t)$.

Proof. If the orientation of $M^{2}$ reverses, then $\lambda$ and $\eta$ both change sign. If the orientation of $\mathcal{E}$ (i.e. the co-orientation) reverses, then $\lambda$ and the $\mathcal{E}$-conormal $n$ both change sign. If $\gamma$ changes orientation, both $\dot{\gamma}$ and $\eta$ change sign. In all cases, the sign of $\kappa_{s}$ is unchanged.

Let $\left\{e_{1}, e_{2}\right\}$ be a positive orthonormal frame field of $\mathcal{E}$ defined on a domain $U \subset M^{2}$. Then there exists a unique 1-form $\omega$ on $U$ such that

$$
D_{X} e_{1}=-\omega(X) e_{2}, \quad D_{X} e_{2}=\omega(X) e_{1} \quad(X \in T U),
$$


which is called the connection form with respect to the frame $\left\{e_{1}, e_{2}\right\}$. The exterior derivative $d \omega$ does not depend on the choice of a positive frame $\left\{e_{1}, e_{2}\right\}$ and gives a (globally defined) 2-form on $M^{2}$. By the definition of $\omega$, we have

$$
d \omega=K d \hat{A}= \begin{cases}K d A & \text { on } M_{+}, \\ -K d A & \text { on } M_{-},\end{cases}
$$

where $K$ is the Gaussian curvature of the first fundamental form $d s^{2}$. When $M^{2}$ is compact, the integration

$$
\chi_{\mathcal{E}}:=\frac{1}{2 \pi} \int_{M^{2}} K d \hat{A}=\frac{1}{2 \pi} \int_{M^{2}} d \omega
$$

is an integer called the Euler number of $\mathcal{E}$.

\section{Peaks and the interior angles between singular curves}

To formulate our generalized Gauss-Bonnet formula, we define further singularities (which is the essentially the same definition as in [SUY]).

Definition 2.1. (Peaks) A singular point $p \in M^{2}$ (which is not an $A_{2}$-point) is called a peak if there exists a coordinate neighborhood $(U ; u, v)$ of $p$ such that:

(1) there are no singular points other than $A_{2}$-points on $U \backslash\{p\}$;

(2) the rank of the linear map $\psi_{p}: T_{p} M^{2} \rightarrow \mathcal{E}_{p}$ at $p$ is equal to 1 ; and

(3) the singular set in $U$ consists of finitely many (possibly empty) $C^{1}$-regular curves starting from $p$. (If such a set of regular curves is empty, the peak $p$ is an isolated singular point.)

If a peak is a non-degenerate singular point, it is called a non-degenerate peak. The singular set $\Sigma$ is said to admit at most peaks if it consists of $A_{2}$-points and peaks.

Let $U$ be a sufficiently small neighborhood of a peak $p$ and $\sigma_{1}, \sigma_{2}$ two singular curves in $U$ starting at $p$. A domain $\Omega$ satisfying the following two conditions is called a singular sector at $p$ :

(1) the boundary of $\Omega \cap U$ consists of $\sigma_{1}, \sigma_{2}$ and the boundary of $U$;

(2) there are no singular points in $\Omega$.

If the peak $p$ is isolated, we also call the domain $\Omega=U \backslash\{p\}$ a singular sector. If $\Omega$ is a singular sector at $p$, the whole of $\Omega$ is contained in $M_{+}$or $M_{-}$. When $\Omega \subset M_{+}$(respectively $\Omega \subset M_{-}$), it is called a positive (negative) singular sector. If there are more than two singular sectors, the number of positive sectors is equal to the number of negative sectors at each peak.

Swallowtails (or, more generally, $A_{3}$-points) are examples of non-degenerate peaks, which have two singular sectors. There are singular points which are not peaks. Typical examples are cone-like singularities which appear in rotationally symmetric surfaces in $\mathbb{R}^{3}$ of positive constant Gaussian curvature. However, since generic fronts (in the local sense) have only cuspidal edges and swallowtails, the set of fronts which admit at most peaks covers a sufficiently wide class of fronts.

Example 2.2. (A double swallowtail [SUY, Example 1.11]) A double swallowtail (or a cuspidal beaks) is a singular point which is $\mathcal{A}$-equivalent to the $C^{\infty}$-map germ

$$
f_{\mathrm{DS}}(u, v):=\left(2 u^{3}-u v^{2}, 3 u^{4}-u^{2} v^{2}, v\right)
$$




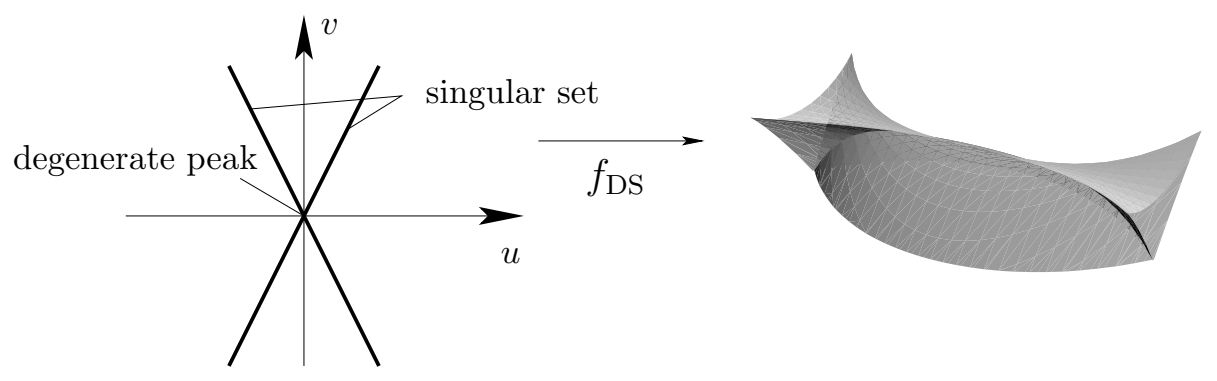

FIGURE 3. A double swallowtail (Example 2.2).

at $(u, v)=(0,0)$, see Figure 3 . Then

$$
v_{\mathrm{DS}}=\frac{1}{\sqrt{1+4 u^{2}\left(1+u^{2} v^{2}\right)}}\left(-2 u, 1,-2 u^{2} v\right)
$$

gives the unit normal vector of $f_{\mathrm{DS}}$. It can be easily checked that $f_{\mathrm{DS}}$ is a front. The signed area density function is $\lambda=\left(v^{2}-6 u^{2}\right) \sqrt{1+4 u^{2}\left(1+u^{2} v^{2}\right)}$, and then the singular set is $\Sigma=\{v=\sqrt{6} u\} \cup\{v=-\sqrt{6} u\}$. In particular, the origin is a degenerate peak, whose neighborhood is divided into four singular sectors (two of them are positive).

Example 2.3. (A cuspidal lips) A cuspidal lips is a singular point which is defined by

$$
f_{\mathrm{CL}}(u, v)=\left(u^{3}+u v^{2}, 3 u^{4}+u^{2} v^{2}, v\right)
$$

see Figure 4, left-hand side. Then

$$
v_{\mathrm{CL}}(u, v)=\frac{1}{\sqrt{1+u^{2}+16 u^{4} v^{2}}}\left(-u,-1,4 u^{2} v\right)
$$

gives the unit normal vector of $f_{\mathrm{CL}}$. It can be easily checked that $f_{\mathrm{CL}}$ is a front. The singular set is the origin, this is an example of a degenerate peak without singular $A_{2}$-curves. (Useful criteria for cuspidal lips and beaks are given in [IST].)

Example 2.4. The tangential developable of the space curve $t \mapsto\left(t^{3}, t^{4}, t^{5}\right)$ is given by

$$
f(t, u):=\left(t^{3}+3 u, t^{4}+4 t u, t^{5}+5 t^{2} u\right) .
$$

Then $(0,0)$ is a non-degenerate peak which is not an $A_{3}$-point (see Figure 4, right-hand side). Ishikawa [I] showed that the tangential developables of the space curves of the form

$$
\gamma(t)=\left(t^{3} a(t), t^{4} b(t), t^{5} c(t)\right) \quad(a(0) b(0) c(0) \neq 0)
$$

at $t=0$ are $\mathcal{A}$-equivalent to this example, where $a(t), b(t), c(t)$ are $C^{\infty}$-functions.

Example 2.5. (The Scherbak surface) The Scherbak surface is a singular point which is defined by

$$
f_{\mathrm{SB}}(u, v)=\left(u^{3}+u^{2} v, 6 u^{5}+5 u^{4} v, v\right),
$$




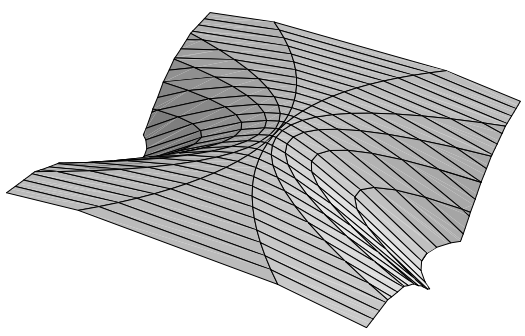

A cuspidal lips

(Example 2.3)

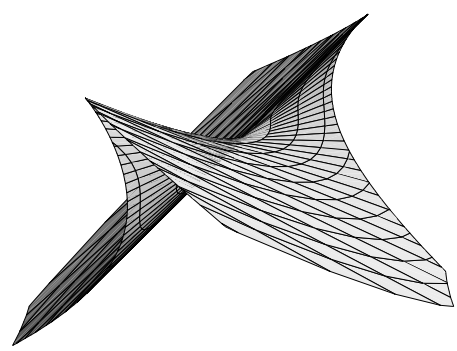

(Example 2.4)

FIGURE 4. A cuspidal lips and Example 2.4.

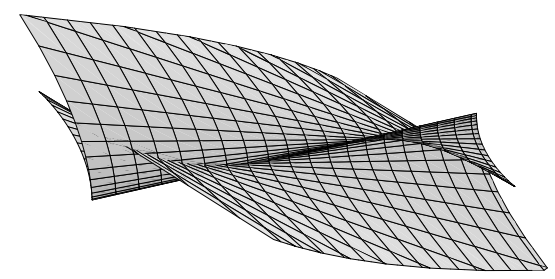

FIGURE 5. The Scherbak surface (Example 2.5).

see Figure 5. Then

$$
\nu_{\mathrm{SB}}(u, v)=\frac{1}{\sqrt{1+100 u^{4}+25 u^{8}}}\left(10 u^{2},-1,-5 u^{4}\right)
$$

gives the unit normal vector of $f_{\mathrm{SB}}$. It can be easily checked that $f_{\mathrm{SB}}$ is a frontal (see the introduction for the definition). The singular set is two transversal lines $\{u=0\} \cup$ $\{3 u+2 v=0\}$. The Scherbak surface is investigated in $[\mathbf{S}, \mathbf{I}, \mathbf{C I}]$.

In this section, we fix a co-oriented coherent tangent bundle $(\mathcal{E},\langle\cdot, \cdot\rangle, D, \psi)$ on an oriented manifold $M^{2}$. Throughout this section, we assume the singular set $\Sigma$ consists of at most peak singularities.

In the following discussion, we fix an arbitrary Riemannian metric $g$ on $M^{2}$. Since the first fundamental form $d s^{2}=\psi^{*}\langle\cdot, \cdot\rangle$ degenerates on $\Sigma$, it is useful to use such a metric $g$ to investigate the property of the singular set $\Sigma$. Then there exists a $(1,1)$-tensor field $I$ on $M^{2}$ such that

$$
d s^{2}(X, Y)=g(I X, Y) \quad\left(X, Y \in T_{p} M^{2}, p \in M^{2}\right) .
$$

We fix a singular point $p \in \Sigma$. Since $\Sigma$ only admits at most peaks, the kernel of $\psi_{p}$ is one dimensional. Thus, only one of the eigenvalues of $I_{p}: T_{p} M^{2} \rightarrow T_{p} M^{2}$ vanishes. So there exists a neighborhood $V$ of $p$ such that $I_{q}$ has two distinct eigenvalues $0 \leq \lambda_{1}(q)<\lambda_{2}(q)$ for each $q \in V$. Since the eigenvectors of these two eigenvalues $\lambda_{1}(q), \lambda_{2}(q)$ depend smoothly on $q \in V$, there exists a coordinate neighborhood $(U ; u, v)$ of $p$ such that $U \subset V$ and 
the $u$-curves (respectively the $v$-curves) give the $\lambda_{1}$-eigendirections (respectively the $\lambda_{2}$ eigendirections) of $I$ on $U$. We call such a local coordinate system $(U ; u, v)$ a $g$-coordinate system at the singular point $p$.

PROPOSITION 2.6. Let $(U ; u, v)$ be a g-coordinate system at a peak $p$, and $\gamma(t)(0 \leq t<1)$ a $C^{1}$-regular curve on $U$ emanating from $p$ such that:

(1) $\dot{\gamma}(0)=d \gamma /\left.d t\right|_{t=0}$ is not a null vector; or

(2) $\gamma$ is a singular curve.

Then there exists a limit

$$
\Psi_{\gamma}:=\lim _{t \rightarrow+0} \frac{\psi(\dot{\gamma}(t))}{|\psi(\dot{\gamma}(t))|} \in \mathcal{E}_{p}
$$

We call this limit vector $\Psi_{\gamma}$ the $\mathcal{E}$-initial vector of $\gamma$ at $p$.

Proof. If $\dot{\gamma}(0)$ is not a null vector, the assertion is obvious. So we may assume that $\gamma$ is a singular curve such that $\dot{\gamma}(0)$ is a null vector. We fix a $g$-coordinate system $(U ; u, v)$ at the peak $p$, and write

$$
\gamma(t)=(u(t), v(t)) \quad(0 \leq t<1) .
$$

Since $\gamma$ is a singular curve, $\left(\psi_{u}:=\right) \psi(\partial / \partial u)$ vanishes on $\gamma$. So we have

$$
\psi(\dot{\gamma}(t))=\dot{v} \psi_{v}(\gamma(t)) \quad(0<t<1),
$$

where $\psi_{v}(q):=\psi_{q}(\partial / \partial v)$ for $q \in U$. Since $\psi_{v}(p) \neq 0$ by the definition of the $g$-coordinate system, we have

$$
\lim _{t \rightarrow+0} \frac{\psi(\dot{\gamma})}{|\psi(\dot{\gamma})|}=\left(\lim _{t \rightarrow+0} \operatorname{sgn} \dot{v}\right) \frac{\psi_{v}(p)}{\left|\psi_{v}(p)\right|} .
$$

Since $\gamma(t)(t>0)$ consists of $A_{2}$-points, we have $\dot{v} \neq 0$ (see the following Proposition 2.13). Hence, the sign of $\dot{v}(t)$ never changes on $t>0$ and then the limit of $\operatorname{sgn}(\dot{v})$ exists, which proves the assertion.

Definition 2.7. Let $(U ; u, v)$ be a local coordinate system centered at a peak $p$ and $\gamma_{j}(t)$ $(0 \leq t<1, j=1,2)$ two $C^{1}$-regular curves in $U$ emanating from $p$ satisfying the assumption of Proposition 2.6. (We might not choose $(u, v)$ to be a $g$-coordinate system here.) Then the angle

$$
\arccos \left(\left\langle\Psi_{\gamma_{1}}, \Psi_{\gamma_{2}}\right\rangle\right) \in[0, \pi]
$$

is called the angle between the initial vectors of $\gamma_{1}, \gamma_{2}$.

Now, we define the interior angle of a singular sector. While it may take a value greater than $\pi$, we have to divide the singular sector into subsectors such that the 'interior angle' does not exceed $\pi$.

First, we assume that $\Omega$ is bounded by two singular curves $\sigma_{0}$ and $\sigma_{1}$. Then there exist a positive integer $n$ and a sequence of $C^{1}$-regular curves starting at $p$

$$
\sigma_{0}=\gamma_{0}, \gamma_{1}, \ldots, \gamma_{n}=\sigma_{1}
$$


in $\Omega$ satisfying the assumption of Proposition 2.6 such that:

- $\gamma_{0}, \ldots, \gamma_{n}$ do not intersect each other in $\Omega$.

- For each $j=1, \ldots, n$, there exists a sector domain $\omega_{j} \subset \Omega$ bounded by $\gamma_{j-1}$ and $\gamma_{j}$ which does not intersect $\gamma_{k}$ for each $k \neq j-1, j$.

- If $n \geq 2,\left\{\dot{\gamma}_{j-1}(0), \dot{\gamma}_{j}(0)\right\}$ is linearly independent and positively oriented for each $j=1, \ldots, n$.

In the following Remark 2.8, we give an explicit way to find $\left\{\gamma_{j}\right\}$.

Next, we assume that the peak $p$ is an isolated singular point. In this case, there are no singular curves which bound the sector $\Omega$, but we can take a sequence $\left\{\gamma_{0}, \gamma_{1}, \gamma_{2}\right\}$ satisfying (2.1)-(2.3), and we set $\gamma_{3}=\gamma_{0}$. See Case 6 in Remark 2.8.

In both cases, the interior angle of the singular sector $\Omega$ is defined as

$$
\arccos \left(\left\langle\Psi_{\gamma_{0}}, \Psi_{\gamma_{1}}\right\rangle\right)+\arccos \left(\left\langle\Psi_{\gamma_{1}}, \Psi_{\gamma_{2}}\right\rangle\right)+\cdots+\arccos \left(\left\langle\Psi_{\gamma_{n-1}}, \Psi_{\gamma_{n}}\right\rangle\right) .
$$

Remark 2.8. There are six possibilities for $\Omega$ as in Figure 6 .

Case 1: $\dot{\sigma}_{0}(0)=k \dot{\sigma}_{1}(0)$ where $k>0$ and $\Omega$ does not contain the direction of $-\dot{\sigma}_{0}(0)$. In this case, we cannot take any interpolation, that is, we must take $n=1$.

Case 2: $\dot{\sigma}_{0}(0)$ and $\dot{\sigma}_{1}(0)$ are transversal and $\Omega$ does not contain the directions of $-\dot{\sigma}_{0}(0)$ and $-\dot{\sigma}_{1}(0)$. In this case, we do not need interpolation, that is, we may take $n=1$.

Case 3: $\dot{\sigma}_{0}(0)=-k \dot{\sigma}_{1}(0)$ where $k>0$. In this case, we need an interpolation, namely, we may take $n=2$.

Case 4: $\dot{\sigma}_{0}(0)$ and $\dot{\sigma}_{1}(0)$ are transversal and $\Omega$ contains the directions of $-\dot{\sigma}_{0}(0)$ and $-\dot{\sigma}_{1}(0)$. In this case, we need an interpolation, namely, we may take $n=2$.

Case 5: $\dot{\sigma}_{0}(0)=k \dot{\sigma}_{1}(0)$, where $k>0$ and $\Omega$ contains the directions of $-\dot{\sigma}_{0}(0)$. In this case, we need two interpolations, namely, we may take $n=3$.

Case 6: The peak is an isolated singular point. In this case, we need three curves.

Later, at the end of this section, we prove the following.

THEOREM A. Let $p \in M^{2}$ be a peak of a coherent tangent bundle $(\mathcal{E},\langle\cdot, \cdot\rangle, D, \psi)$ on $M^{2}$. Then the sum $\alpha_{+}(p)$ (respectively $\alpha_{-}(p)$ ) of all interior angles of positive (respectively negative) singular sectors at $p$ satisfies

$$
\begin{gathered}
\alpha_{+}(p)+\alpha_{-}(p)=2 \pi, \\
\alpha_{+}(p)-\alpha_{-}(p) \in\{2 \pi, 0,-2 \pi\} .
\end{gathered}
$$

Definition 2.9. A peak $p$ is called positive, null, negative according to the sign of $\alpha_{+}(p)-$ $\alpha_{-}(p)$.

Remark 2.10. The formulas (2.5) and (2.6) are intrinsic versions of (2.2) and (2.3) in [SUY], respectively. 


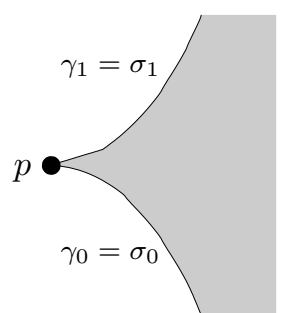

Case 1

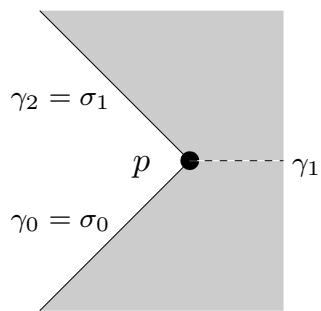

Case 4

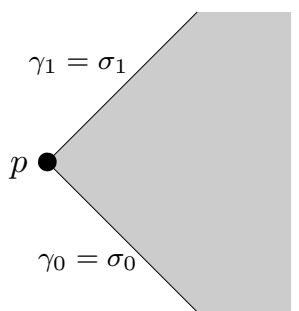

Case 2

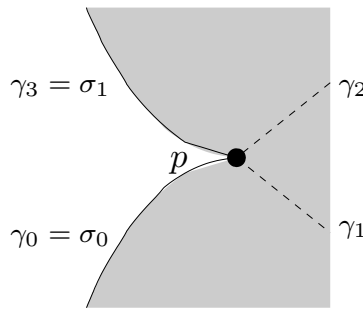

Case 5

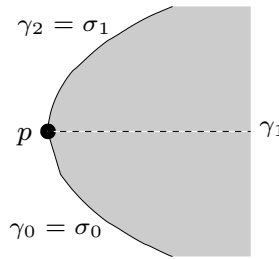

Case 3

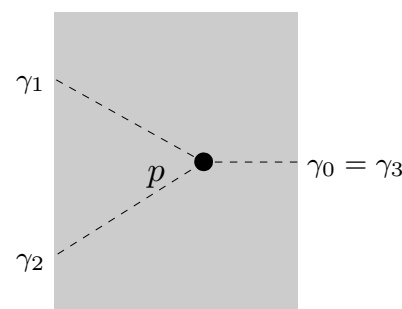

Case 6

FIGURE 6. Six possibilities for $\Omega$.

For our further analysis of the singular curvature near a peak, we prepare the following assertion which is the intrinsic version of [SUY, Proposition 1.12].

PROPOSITION 2.11. (Boundedness of the singular curvature measure) Take a singular curve $\gamma:[0, \varepsilon) \rightarrow M^{2}$ starting from a peak $p$ such that $\gamma(t)$ is an $A_{2}$-point for each $t>0$. Then the singular curvature measure $\kappa_{s} d \tau$ is continuous on $[0, \varepsilon)$, where $d \tau$ is the arclength measure with respect to the first fundamental form $d s^{2}$.

Proof. We can take a $g$-coordinate system $(u, v)$ such that $\partial / \partial u$ is the null vector field on $\gamma$. For the sake of simplicity, we set

$$
\psi_{u}:=\psi(\partial / \partial u), \quad \psi_{v}:=\psi(\partial / \partial v), \quad \dot{\hat{\gamma}}(t):=\psi(\dot{\gamma}(t)) \quad\left(\cdot=\frac{d}{d t}\right) .
$$

In such a coordinate system, $\psi_{u}=0$ and $D_{t} \psi_{u}=0$ hold on $\gamma$. Then

$$
\dot{\hat{\gamma}}=\dot{v} \psi_{v}, \quad D_{t} \dot{\hat{\gamma}}=\ddot{v} \psi_{v}+\dot{v} D_{t} \psi_{v} .
$$

Hence,

$$
\kappa_{s}= \pm \frac{\mu\left(\dot{\hat{\gamma}}, D_{t} \dot{\hat{\gamma}}\right)}{|\dot{\hat{\gamma}}|^{3}}= \pm \frac{\mu\left(\psi_{v}, D_{t} \psi_{v}\right)}{|\dot{v}|\left|\psi_{v}\right|^{3}} .
$$

Since $d \tau=|\dot{\hat{\gamma}}| d t=|\dot{v}|\left|\psi_{v}\right| d t$ and $\psi_{v} \neq 0$,

$$
\kappa_{s} d \tau= \pm \frac{\mu\left(\psi_{v}, D_{t} \psi_{v}\right)}{\left|\psi_{v}\right|^{2}} d t
$$

is bounded. 
Then we can state generalized Gauss-Bonnet formulas.

THEOREM B. Let $M^{2}$ be a compact oriented 2-manifold and $\mathcal{E}$ a coherent tangent bundle whose singular set $\Sigma$ admits at most peaks. Then

$$
\begin{gathered}
\left(\chi_{\mathcal{E}}=\right) \frac{1}{2 \pi} \int_{M^{2}} K d \hat{A}=\chi\left(M_{+}\right)-\chi\left(M_{-}\right)+\# P_{+}-\# P_{-}, \\
2 \pi \chi\left(M^{2}\right)=\int_{M^{2}} K d A+2 \int_{\Sigma} \kappa_{s} d \tau
\end{gathered}
$$

hold, where $d \tau$ is the arclength measure on the singular set and \# $P_{+}, \# P_{-}$are the numbers of positive and negative peaks, respectively, defined in Definition 2.9.

The identities (2.8) and (2.9) are generalizations of (1) and (2) in the introduction, respectively. The proof is given in Section 4.

It should be remarked that the integral $\int_{\Sigma} \kappa_{S} d \tau$ is well defined by Proposition 2.11. In [SUY], the authors did not state Theorem B intrinsically as above. The two formulas (2.8) and (2.9) are not only generalizations of the formulas given in the introduction, but also those in [SUY].

To prove Theorem A, we need a tool to measure the interior angle between 'curves' starting at a peak. We define a class of curves such that the interior angles are well defined.

Definition 2.12. (Admissible curves) A curve $\sigma(t)(t \in[a, b])$ on $U$ is called admissible if it satisfies one of the following conditions:

(1) $\sigma$ is a $C^{1}$-regular curve such that $\sigma((a, b))$ does not contain a peak, and the tangent vector $\dot{\sigma}(t)(t \in[a, b])$ is transversal to the singular direction and the null direction if $\sigma(t) \in \Sigma$;

(2) the set $\sigma([a, b])$ is contained in a singular set $\Sigma$ and the set $\sigma((a, b))$ does not contain a peak.

Next, we prove the following assertion, which plays a crucial role in the proof of Theorem A.

PROPOSITION 2.13. Suppose that $p$ is a peak. Then there exists a g-coordinate system $(U ; u, v)$ such that each admissible curve $\gamma(t)(t \geq 0)$ starting at $p$ does not have velocity vector $\dot{\gamma}(t)(t>0)$ parallel to the $u$-axis on $U$. In particular, $\gamma(t)(t>0)$ never meets the u-axis.

Proof. We fix a $g$-coordinate system $(U ; u, v)$ at $p$. By definition, for any admissible curve $\sigma$ in $U$ which is not a singular curve, $\dot{\sigma}(t)$ is never parallel to the $u$-axis whenever $U$ is sufficiently small, because $\dot{\sigma}(0)$ is transversal to the $u$-direction. So it is sufficient to consider only singular curves. We now fix a singular curve $\gamma(t)(t \geq 0)$ on $U$ such that $\gamma(0)=p$. Since the number of singular curves starting at $p$ is finite, it is sufficient to show that there exists a (sufficiently small) $\varepsilon>0$ such that $\dot{\gamma}(t)$ will never be parallel to the $u$-axis on $(0, \varepsilon]$. (The second assertion immediately follows from the mean value theorem.) If $\dot{\gamma}(0)$ is transversal to the $u$-axis, it is obvious. So we may assume that $\dot{\gamma}(0)$ is proportional to the $u$-axis. Then $\gamma$ can be expressed as a graph $v=F(u)(0 \leq u \leq \delta)$. If there exists $c \in(0, \delta)$ such that $d F / d u$ vanishes at $u=c$, then we have a contradiction since the null direction $\partial / \partial u$ is proportional to the singular direction. (On a $g$-coordinate system, the null direction always points in the $u$-direction on each singular curve, by its definition.) 


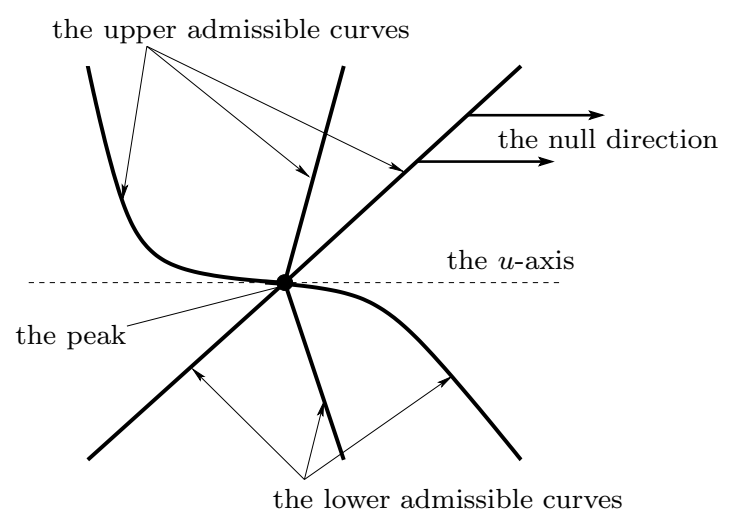

FIGURE 7. Upper and lower admissible curves.

We divide the set of admissible curves starting at the peak $p$ into the following two classes (see Figure 7).

(i) The admissible curves which lie in the upper half-plane of the $g$-coordinate system are called the upper admissible curves.

(ii) The admissible curves which lie in the lower half-plane of the $g$-coordinate system are called the lower admissible curves.

PROPOSITION 2.14. These two classes of the admissible curves starting from $p$ are independent of the choice of Riemannian metric $g$ on $M^{2}$.

Proof. Let $h$ be another Riemannian metric on $M^{2}$. Then $(1-t) g+t h(t \in[0,1])$ gives the deformation between two metrics. During the deformation of the metric, each admissible curve never meets the $u$-axis for a fixed sufficiently small neighborhood of $p$.

Note that admissible curves have initial vectors, by Proposition 2.6. Now, we prove that the angle between two admissible curves at a peak are determined by their classes.

PROPOSITION 2.15. Let $\gamma_{j}(t)(j=0,1)$ be two admissible curves starting at a peak $p$. Then the $\mathcal{E}$-initial vector $\Psi_{\gamma_{1}}$ is equal to $\Psi_{\gamma_{2}}$ (respectively $-\Psi_{\gamma_{2}}$ ) if and only if $\left\{\gamma_{1}, \gamma_{2}\right\}$ are in the same class (respectively distinct classes).

Proof. Take a $g$-coordinate system $(U ; u, v)$ at $p$. If $\dot{\gamma}_{j}(0)$ is not a null vector, then

$$
\Psi_{\gamma_{j}}=\operatorname{sgn}\left(\dot{v}_{j}(0)\right) \frac{\psi_{p}(\partial / \partial v)}{\left|\psi_{p}(\partial / \partial v)\right|} \quad\left(\gamma_{j}(t)=\left(u_{j}(t), v_{j}(t)\right)\right) .
$$

On the other hand, if $\gamma_{j}(t)$ is a singular curve with the null vector $\dot{\gamma}_{j}(0)$, as seen in the proof of Proposition 2.6, we obtain

$$
\Psi_{\gamma_{j}}=\left(\lim _{t \rightarrow+0} \operatorname{sgn} \dot{v}_{j}(t)\right) \frac{\psi_{p}(\partial / \partial v)}{\left|\psi_{p}(\partial / \partial v)\right|} .
$$

These two formulas for the $\mathcal{E}$-initial vector $\Psi_{\gamma_{j}}$ prove the assertion. 

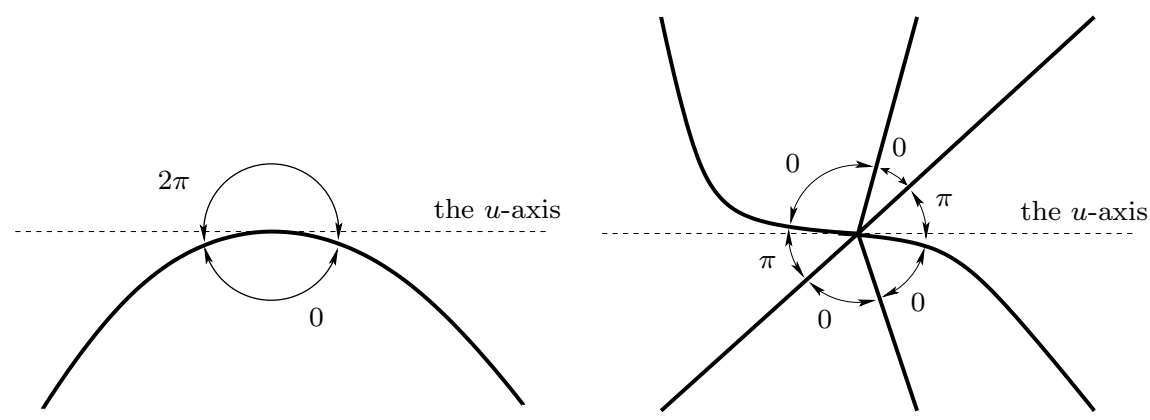

FIGURE 8. Interior angles.

COROLlary 2.16. Let $(U ; u, v)$ be a g-coordinate system at a peak $p$, and $\Omega$ a singular sector. Then the interior angle $\alpha_{\Omega}$ of $\Omega$ (defined in (2.4)) is given by

$$
\alpha_{\Omega}= \begin{cases}2 \pi & \text { if } \Omega \cup\{p\} \text { contains the closed upper-half or the closed lower-half uv-plane } \\ & \text { near } p, \\ 0 \quad & \text { if } \Omega \text { is contained in the open upper-half or the open lower-half uv-plane, } \\ \pi \quad & \text { otherwise. }\end{cases}
$$

See Figure 8.

Using this corollary, we can easily compute the angle of each singular sector at a peak. For example, the singular set near the peak of the swallowtail $f_{\mathrm{SW}}$ given (1.3) is a parabola in the $u v$-plane. So both of the singular curves starting at the origin are in the same class. If we take the unit normal vector $v_{\mathrm{SW}}=\left(1, u, u^{2}\right) / \sqrt{1+u^{2}+u^{4}}$ of $f_{\mathrm{SW}}$, then the positive sector is the upper-half domain of the parabola and its interior angle is $2 \pi$.

On the other hand, near the peak of the double swallowtail as in Example 2.2, the singular set can be taken to be two lines transversally intersecting at the origin, i.e. consisting of four rays. Since the null direction is $\partial / \partial u$ on the singular set, they are divided into two classes consisting of two rays. The interior angles of positive sectors are both zero and the negative sectors are both $\pi$ (see Figure 3).

In the case of an isolated peak as in Example 2.3, namely a cuspidal lips, the neighborhood of the origin is the positive or negative sector, and the interior angle is $2 \pi$.

Proof of Theorem A. The singular curves starting at the peak divide the neighborhood of $p$ into sectors consisting of subsets of $M_{+}$or $M_{-}$. However, by Corollary 2.16, there is no contribution of the interior angle of the sector unless it contains the $u$-axis to the $g$-coordinate system, that is, the only two sectors containing the $u$-axis have interior angle $\pi$. Thus, we have

$$
\alpha_{+}+\alpha_{-}=2 \pi
$$

and

$$
\alpha_{-}, \alpha_{+} \in\{0, \pi, 2 \pi\},
$$

which proves the assertion. 


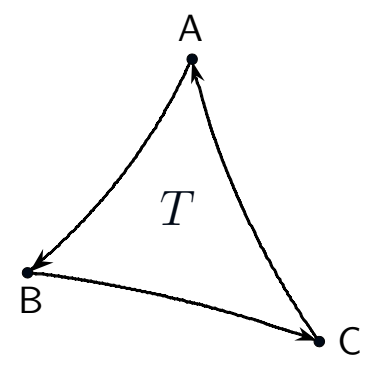

FIGURE 9. An admissible triangle.

\section{A local Gauss-Bonnet formula}

In this section, we state a local Gauss-Bonnet-type theorem for 'admissible' triangles. Let $p$ be a peak and fix a $g$-coordinate system $(U ; u, v)$ at $p$. Let $\sigma(t)$ be an admissible curve (in the sense of Definition 2.12). We define the geometric curvature $\tilde{\kappa}_{g}$ of $\sigma$ as follows:

$$
\tilde{\kappa}_{g}(t)=\left\{\begin{aligned}
\hat{\kappa}_{g}(t) & \text { if } \sigma(t) \in M_{+}, \\
-\hat{\kappa}_{g}(t) & \text { if } \sigma(t) \in M_{-}, \\
\kappa_{S}(t) & \text { if } \sigma(t) \in \Sigma .
\end{aligned}\right.
$$

Here, this (geometric) curvature $\tilde{\kappa}_{g}$ is the geodesic curvature with respect to the orientation of $M^{2}$ which coincides with the curvature $\hat{\kappa}_{g}$ defined by (1.5) on $M_{+}$and is equal to $-\hat{\kappa}_{g}$ on $M_{-}$.

Definition 3.1. (Admissible triangles) Let $\bar{T} \subset U$ be the closure of a simply connected domain $T$ which is bounded by three admissible arcs $\gamma_{1}, \gamma_{2}, \gamma_{3}$. Let A, B and C be the distinct three boundary points of $T$ which are intersections of these three $\operatorname{arcs}$. Then $\bar{T}$ is called an admissible triangle if it satisfies the following three conditions:

(i) $\bar{T}$ admits at most one peak on $\{\mathrm{A}, \mathrm{B}, \mathrm{C}\}$;

(ii) the three interior angles at $A, B$ and $C$ with respect to the metric $g$ are all less than $\pi$;

(iii) if $\gamma_{j}(j=1,2,3)$ is not a singular curve, it is $C^{2}$-regular, namely it is a restriction of a certain open $C^{2}$-regular arc.

We write

$$
\triangle \mathrm{ABC}:=\bar{T}
$$

and call $\{A, B, C\}$ the vertices of the triangle. We also denote by

$$
\widehat{\mathrm{BC}}:=\gamma_{1}, \widehat{\mathrm{CA}}:=\gamma_{2} \text { and } \widehat{\mathrm{AB}}:=\gamma_{3}
$$

the regular arcs whose boundary points are $\{B, C\},\{C, A\}$ and $\{A, B\}$, respectively. We give here the orientation of these three arcs such that the left-hand side is $T$, namely the cyclic order $(\mathrm{A}, \mathrm{B}, \mathrm{C})$ is compatible with respect to the orientation of $M^{2}$, see Figure 9.

We also denote by

$$
\angle A, \angle B \text { and } \angle C
$$


the interior angles (with respect to the first fundamental form $d s^{2}=\psi^{*}\langle\cdot, \cdot\rangle$ ) of the piecewise smooth boundary of $\triangle A B C$ at $A, B$ and $C$, respectively, unless $A, B$ and $C$ are not singular points. On the other hand, if $A$ is a singular point, we set

$$
\angle \mathrm{A}:= \begin{cases}\pi & \text { if the } u \text {-curve passing through } \mathrm{A} \text { separates } \widehat{\mathrm{AB}} \text { and } \widehat{\mathrm{AC}}, \\ 0 & \text { otherwise. }\end{cases}
$$

Similarly we can define $\angle B$ (respectively $\angle C$ ) when $B$ (respectively $C$ ) is a singular point.

Remark 3.2. By Proposition 2.15, $L$ A coincides with the angle of the $\mathcal{E}$-initial vectors between $\widehat{A B}$ and $\widehat{A C}$.

THEOREM 3.3. (The local Gauss-Bonnet formula) Let $(\mathcal{E},\langle\cdot, \cdot\rangle, D, \psi)$ be a coherent tangent bundle and $\triangle \mathrm{ABC}$ an admissible triangle on $M^{2}$. Then the following identity holds:

$$
\angle \mathrm{A}+\angle \mathrm{B}+\angle \mathrm{C}-\pi=\int_{\partial \triangle \mathrm{ABC}} \tilde{\kappa}_{g} d \tau+\int_{\triangle \mathrm{ABC}} K d A+2 \int_{\Sigma \cap(\triangle \mathrm{ABC})^{\circ}} \kappa_{s} d \tau,
$$

where $\Sigma$ is the singular set, $(\triangle \mathrm{ABC})^{\circ}$ (respectively $\partial \triangle \mathrm{ABC}$ ) the interior (the boundary) of the closed domain $\triangle \mathrm{ABC}$, and $K$ is the Gaussian curvature of the metric $d s^{2}=\psi^{*}\langle\cdot, \cdot\rangle$ on $M^{2} \backslash \Sigma$. In particular, if there are no singular points in the interior of the triangle, it holds that

$$
\angle \mathrm{A}+\angle \mathrm{B}+\angle \mathrm{C}-\pi=\int_{\partial \triangle \mathrm{ABC}} \tilde{\kappa}_{g} d \tau+\int_{\triangle \mathrm{ABC}} K d A .
$$

To prove Theorem 3.3, we prepare several lemmas as follows.

LEMmA 3.4. Suppose that $\triangle \mathrm{ABC}$ is contained in $M_{+}$or $M_{-}$. Then (3.3) holds.

Proof. The lemma is exactly the classical Gauss-Bonnet formula with respect to the Riemannian metric $d s^{2}$ on $M^{2} \backslash \Sigma$.

LEMMA 3.5. Let $\triangle \mathrm{ABC}$ be an admissible triangle such that $\mathrm{A}$ is a $A_{2}$-point or a peak, and $\triangle \mathrm{ABC} \backslash\{\mathrm{A}\}$ lies in $M_{+}$(respectively $M_{-}$). Suppose that $\widehat{\mathrm{AB}}$ and $\widehat{\mathrm{BC}}$ are transversal at $\mathrm{B}$. Then (3.3) holds.

Proof. Without loss of generality, we may assume that $\triangle \mathrm{ABC} \backslash\{\mathrm{A}\}$ lies in $M_{+}$. We can take a short extension of the $C^{2}$-regular arc $\widehat{\mathrm{AB}}$ beyond $\mathrm{A}$, and rotate it around $\mathrm{B}$ with respect to the canonical metric $d u^{2}+d v^{2}$ on the $u v$-plane. Then we obtain a smooth one-parameter family of $C^{2}$-regular arcs staring at $\mathrm{B}$. Since $\widehat{\mathrm{AB}}$ and $\widehat{\mathrm{BC}}$ are transversal at $\mathrm{B}$, restricting the image of this family on the triangle $\triangle A B C$, we obtain a family of $C^{2}$-regular curves

$$
\gamma_{\varepsilon}:[0,1] \longrightarrow \triangle \mathrm{ABC} \quad(\varepsilon \in[0,1])
$$

such that (see Figure 10, left-hand side):

(i) $\quad \gamma_{0}$ parameterizes $\widehat{\mathrm{AB}}$ such that $\gamma_{0}(1)=\mathrm{A}$ and $\gamma_{0}(0)=\mathrm{B}$;

(ii) $\gamma_{\varepsilon}(0)=\mathrm{B}$ for all $\varepsilon \in[0,1]$;

(iii) the correspondence $\sigma: \varepsilon \mapsto \gamma_{\varepsilon}(1)$ gives a subarc on $\widehat{A C}$; we set $\mathbf{A}_{\varepsilon}=\gamma_{\varepsilon}(1)$, where $A_{0}=A$. 


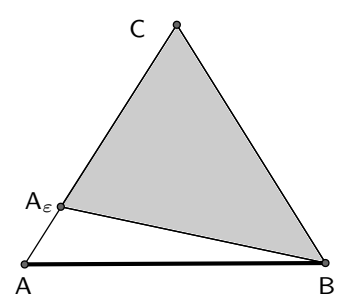

Proofs of Lemmas 3.5 and 3.6

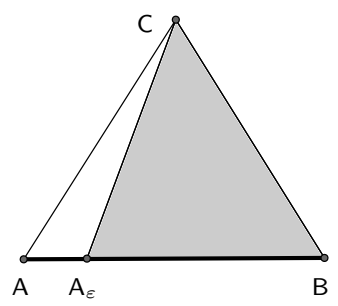

Proof of Lemma 3.7

FIGURE 10. Proofs of Lemmas 3.5, 3.6 and 3.7.

Since $\triangle \mathrm{A}_{\varepsilon} \mathrm{BC}(\varepsilon>0)$ lies in $M_{+}$, it is an admissible triangle. So, applying Lemma 3.4 for $\triangle A_{\varepsilon} B C$, we have

$$
\angle \mathrm{A}_{\varepsilon}+\angle \mathrm{A}_{\varepsilon} \mathrm{BC}+\angle \mathrm{C}-\pi=\int_{\partial \triangle \mathrm{A}_{\varepsilon} \mathrm{BC}} \tilde{\kappa}_{g} d \tau+\int_{\triangle \mathrm{A}_{\varepsilon} \mathrm{BC}} K d A .
$$

By taking the limit as $\varepsilon \rightarrow 0$, we have that

$$
\lim _{\varepsilon \rightarrow+0} \angle \mathrm{A}_{\varepsilon}+\angle \mathrm{B}+\angle \mathrm{C}-\pi=\int_{\partial \triangle \mathrm{ABC}} \tilde{\kappa}_{g} d \tau+\int_{\triangle \mathrm{ABC}} K d A .
$$

Note that since $\triangle \mathrm{ABC}$ is admissible, $\hat{\kappa}_{g}$ is bounded on both of $\widehat{A B}$ and $\widehat{A C}$. On the other hand, by Proposition 2.15 we have

$$
\begin{aligned}
& \lim _{\varepsilon \rightarrow+0} L \mathbf{A}_{\varepsilon} \\
& =\lim _{\varepsilon \rightarrow+0} \arccos \left[\left\langle\dot{\hat{\gamma}}_{\varepsilon}(0), \psi\left(\frac{d \gamma_{s}(0)}{d s}\right)\right\rangle /\left(\left|\dot{\hat{\gamma}}_{\varepsilon}(0)\right|\left|\psi\left(\frac{d \gamma_{s}(0)}{d s}\right)\right|\right)\right]_{s=\varepsilon} \\
& =\arccos \left\langle\Psi_{\gamma_{0}}, \Psi_{\sigma}\right\rangle= \begin{cases}\pi & \text { if the } u \text {-curve passing through } \mathrm{A} \text { separates } \widehat{\mathrm{AB}} \text { and } \widehat{\mathrm{AC}}, \\
0 & \text { otherwise, }\end{cases}
\end{aligned}
$$

where $\dot{\hat{\gamma}}_{\varepsilon}(t)=\psi\left(d \gamma_{\varepsilon}(t) / d t\right)$. This completes the proof.

LEMMA 3.6. Let $\triangle \mathrm{ABC}$ be an admissible triangle such that $\widehat{\mathrm{AB}}$ consists of $A_{2}$-points, and $\triangle \mathrm{ABC} \backslash \widehat{\mathrm{AB}}$ lies in $M_{+}$(respectively in $\left.M_{-}\right)$. Suppose that $\widehat{\mathrm{AB}}$ and $\widehat{\mathrm{BC}}$ are transversal at $\mathrm{B}$. Then (3.3) holds.

Proof. Without loss of generality, we may assume that $\triangle \mathrm{ABC} \backslash \widehat{\mathrm{AB}}$ lies in $M_{+}$. Since $\mathrm{A}$ and $\mathrm{B}$ are $A_{2}$-points, by the same method as in Lemma 3.5 there is a family of $C^{2}$-regular curves

$$
\gamma_{\varepsilon}:[0,1] \longrightarrow \triangle \mathrm{ABC} \quad(\varepsilon \in[0,1])
$$

such that:

(i) $\gamma_{0}$ parametrizes $\widehat{\mathrm{AB}}$ such that $\gamma_{0}(1)=\mathrm{A}$ and $\gamma_{0}(0)=\mathrm{B}$,

(ii) $\gamma_{\varepsilon}(0)=\mathrm{B}$ for all $\varepsilon \in[0,1]$;

(iii) the correspondence $\varepsilon \mapsto \gamma_{\varepsilon}(1)$ gives a subarc on $\widehat{A C}$; we set $A_{\varepsilon}=\gamma_{\varepsilon}(1)$, where $A_{0}=A$. 
Since $\triangle \mathrm{A}_{\varepsilon} \mathrm{BC}(\varepsilon>0)$ lies in $M_{+}$, it is an admissible triangle. So, applying Lemma 3.5 for $\triangle \mathrm{A}_{\varepsilon} \mathrm{BC}$, we have

$$
\angle \mathrm{A}_{\varepsilon}+\angle \mathrm{A}_{\varepsilon} \mathrm{BC}+\angle \mathrm{C}-\pi=\int_{\partial \triangle \mathrm{A}_{\varepsilon} \mathrm{BC}} \tilde{\kappa}_{g} d \tau+\int_{\triangle \mathrm{A}_{\varepsilon} \mathrm{BC}} K d A .
$$

By taking the limit as $\varepsilon \rightarrow 0$, we have the assertion just by the same argument as in the proof of the previous lemma.

LEMMA 3.7. Let $\mathrm{A}$ be a peak of an admissible triangle $\triangle \mathrm{ABC}$ such that $\widehat{\mathrm{AB}}$ is a singular curve starting from $\mathrm{A}$, and $\triangle \mathrm{ABC} \backslash \widehat{\mathrm{AB}}$ is contained completely in $M_{+}$or in $M_{-}$. Suppose that $\widehat{\mathrm{BC}}$ and $\widehat{\mathrm{CA}}$ are transversal at $\mathrm{C}$. Then (3.3) holds.

Proof. We may assume that $\triangle \mathrm{ABC}$ lies in $\bar{M}_{+}$. We can take a short extension of the $C^{2}$ regular arc $\widehat{A C}$ beyond $A$, and rotate it around $C$ with respect to the canonical metric $d u^{2}+d v^{2}$ on the $u v$-plane. Then we get a smooth one-parameter family of $C^{2}$-regular arcs staring at $C$. Since $\widehat{B C}$ and $\widehat{A C}$ are transversal at $C$, restricting the image of this family on the triangle $\triangle \mathrm{ABC}$, we obtain a family of $C^{2}$-regular curves

$$
\gamma_{\varepsilon}:[0,1] \longrightarrow \triangle \mathrm{ABC}, \quad(\varepsilon \in[0,1])
$$

such that (see Figure 10, right-hand side):

(i) $\quad \gamma_{0}$ parametrizes $\widehat{A C}$ such that $\gamma_{0}(1)=\mathrm{A}$ and $\gamma_{0}(0)=\mathrm{C}$;

(ii) $\gamma_{\varepsilon}(0)=\mathrm{C}$ for all $\varepsilon \in[0,1]$;

(iii) the correspondence $\varepsilon \mapsto \gamma_{\varepsilon}(1)$ gives a subarc on $\widehat{A B}$; we set $\mathbf{A}_{\varepsilon}=\gamma_{\varepsilon}(1)$, where $\mathbf{A}_{0}=\mathbf{A}$.

Since $\widehat{A C}$ is admissible, its tangential vector at $A$ does not point in the null direction. Hence, the tangential vector of $\widehat{A_{\varepsilon}} C$ at $A_{\varepsilon}$ does not point in the null direction for sufficiently small $\varepsilon>0$ and $\triangle A_{\varepsilon} B C$ is an admissible triangle. Applying Lemma 3.6 for $\triangle A_{\varepsilon} B C$, we have

$$
\angle \mathrm{A}_{\varepsilon}+\angle \mathrm{A}_{\varepsilon} \mathrm{CB}+\angle \mathrm{B}-\pi=\int_{\partial \triangle \mathrm{A}_{\varepsilon} \mathrm{BC}} \tilde{\kappa}_{g} d \tau+\int_{\triangle \mathrm{A}_{\varepsilon} \mathrm{BC}} K d A .
$$

By Proposition 2.11 and the Lebesgue convergence theorem, the limit

$$
\int_{\partial \triangle \mathrm{ABC}} \tilde{\kappa}_{g} d \tau=\lim _{\varepsilon \rightarrow+0} \int_{\partial \triangle \mathrm{A}_{\varepsilon} \mathrm{BC}} \tilde{\kappa}_{g} d \tau
$$

exists. On the other hand, since the property that the $u$-direction $\partial / \partial u$ at $\mathrm{A}_{\varepsilon}$ is inward or outward is common in $\varepsilon \in(0,1]$ (cf. the proof of Theorem 2.13), $\angle \mathbf{A}_{\varepsilon}$ is common in $\varepsilon$. Thus, we have

$$
\angle \mathrm{A}=\lim _{\varepsilon \rightarrow+0} \angle \mathrm{A}_{\varepsilon}= \begin{cases}\pi & \text { if the } u \text {-curve separates } \widehat{\mathrm{AB}} \text { and } \widehat{\mathrm{AC}}, \\ 0 & \text { otherwise. }\end{cases}
$$

This proves the assertion.

LEMMA 3.8. Let $\triangle \mathrm{ABC}$ be an admissible triangle such that $\mathrm{A}$ is a regular point or at most a peak singular point. Suppose that there is at most one singular curve in $\triangle \mathrm{ABC}$ starting at A from the null direction. Then (3.3) holds. 


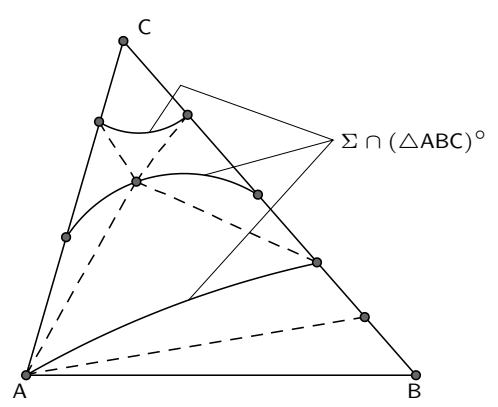

FIGURE 11. A proof of Lemma 3.8.

Proof. By a suitable division, the triangle decomposed into admissible triangles which satisfy the conditions as in Lemmas 3.4-3.7. Then the formula is proved, since the geometric curvature on each edge consists of a singular curve that is duplicated. See Figure 11.

LEMMA 3.9. Let $\mathrm{A}$ be a peak of an admissible triangle $\triangle \mathrm{ABC}$ such that $\widehat{\mathrm{CA}}$ is not a singular curve, and $\widehat{\mathrm{BC}}$ and $\widehat{\mathrm{CA}}$ are transversal at $\mathrm{C}$. Suppose that either $\widehat{\mathrm{AB}}$ or $\widehat{\mathrm{AC}}$ is not a singular curve. Then (3.3) holds.

Proof. The proof is almost parallel to that of Lemma 3.7 (instead of Lemma 3.6, we apply Lemma 3.8): without loss of generality, we may assume that $\widehat{A B}$ is a singular curve. Then $\widehat{A C}$ is a $C^{2}$-regular arc. We can take a short extension of $\widehat{A C}$ over $\mathrm{A}$, and rotate it around $\mathrm{C}$ with respect to the metric $d u^{2}+d v^{2}$. Then we obtain a smooth one-parameter family of arcs starting at $C$. Since $\widehat{B C}$ and $\widehat{A C}$ are transversal at $C$ by the assumption of the lemma, restricting the image of this family to the triangle $\triangle A B C$, we obtain a family of $C^{2}$-regular curves

$$
\gamma_{\varepsilon}:[0,1] \longrightarrow \triangle \mathrm{ABC} \quad(\varepsilon \in[0,1])
$$

such that:

(i) $\gamma_{0}$ parametrizes $\widehat{A C}$ such that $\gamma_{0}(1)=\mathrm{A}$ and $\gamma_{0}(0)=\mathrm{C}$;

(ii) $\gamma_{\varepsilon}(0)=\mathrm{C}$ for all $\varepsilon \in[0,1]$;

(iii) the correspondence $\varepsilon \mapsto \gamma_{\varepsilon}(1)$ gives a subarc on $\widehat{A B}$; we set $\mathbf{A}_{\varepsilon}=\gamma_{\varepsilon}(1)$, where $\mathbf{A}_{0}=\mathrm{A}$.

Since $\widehat{A C}$ is admissible, its tangential vector at $A$ does not point in the null direction.

Hence, the tangential vector of $\widehat{A_{\varepsilon}} \mathrm{C}$ at $\mathrm{A}_{\varepsilon}$ does not point in the null direction for sufficiently small $\varepsilon>0$ and $\triangle \mathrm{A}_{\varepsilon} \mathrm{BC}$ is an admissible triangle. Applying Lemma 3.8 for $\triangle \mathrm{A}_{\varepsilon} \mathrm{BC}$, we have

$$
\angle \mathrm{A}_{\varepsilon}+\angle \mathrm{A}_{\varepsilon} \mathrm{CB}+\angle \mathrm{B}-\pi=\int_{\partial \triangle \mathrm{A}_{\varepsilon} \mathrm{BC}} \tilde{\kappa}_{g} d \tau+\int_{\triangle \mathrm{A}_{\varepsilon} \mathrm{BC}} K d A+2 \int_{\Sigma \cap\left(\triangle \mathrm{A}_{\varepsilon} \mathrm{BC}\right)^{\circ}} \kappa_{s} d \tau .
$$

By Proposition 2.11 and the Lebesgue convergence theorem, the limits

$$
\int_{\partial \triangle \mathrm{ABC}} \tilde{\kappa}_{g} d \tau=\lim _{\varepsilon \rightarrow+0} \int_{\partial \triangle \mathrm{A}_{\varepsilon} \mathrm{BC}} \tilde{\kappa}_{g} d \tau,
$$




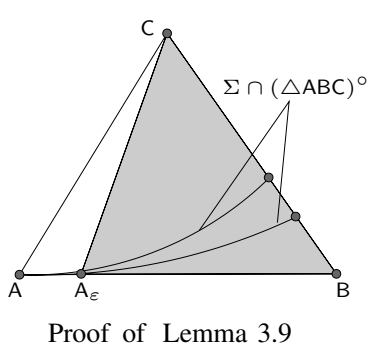

Proof of Lemma 3.9

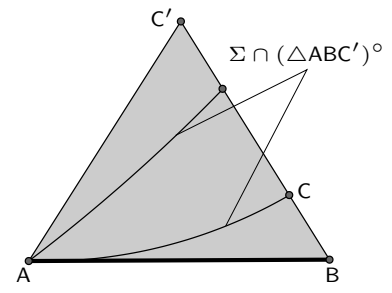

Proof of Theorem 3.3

FIgURE 12. Proofs of Lemma 3.9 and Theorem 3.3.

and

$$
\int_{\Sigma \cap(\triangle \mathrm{ABC})^{\circ}} \kappa_{s} d \tau=\lim _{\varepsilon \rightarrow+0} \int_{\Sigma \cap\left(\triangle \mathrm{A}_{\varepsilon} \mathrm{BC}\right)^{\circ}} \kappa_{s} d \tau
$$

exist. On the other hand, since the property that the $u$-direction $\partial / \partial u$ at $\mathrm{A}_{\varepsilon}$ is inward or outward is common in $\varepsilon \in(0,1]$ (cf. the proof of Theorem 2.13), $\angle A_{\varepsilon}$ is common in $\varepsilon$. Thus, we have

$$
\angle \mathrm{A}=\lim _{\varepsilon \rightarrow+0} \angle \mathrm{A}_{\varepsilon}= \begin{cases}\pi & \text { if the } u \text {-curve separates } \widehat{\mathrm{AB}} \text { and } \widehat{\mathrm{AC}}, \\ 0 & \text { otherwise. }\end{cases}
$$

This proves the assertion.

Proof of Theorem 3.3. As seen in the proof of Lemma 3.8, the given triangle can be divided into small triangles. So, as the only remaining possibility, it is sufficient to consider the case that $\triangle \mathrm{ABC}$ has the following four properties:

(1) $A$ is a peak;

(2) $\widehat{A B}$ and $\widehat{A C}$ are both singular curves which have the same null-velocity vector at $A$;

(3) $\widehat{B C}$ is not a singular curve;

(4) there are no singular points on the inside of the triangle $\triangle A B C$.

Take a new smooth arc $\widehat{\mathrm{BC}^{\prime}}$ such that $\widehat{\mathrm{BC}}$ is a subarc. Then we consider a new admissible triangle $\triangle A B C^{\prime}$, which contains $\triangle A B C$ as a subset, see Figure 12, right-hand side.

In this situation, the two triangles $\triangle A B C^{\prime}$ and $\triangle A C C^{\prime}$ satisfy the assumption of Lemma 3.9. So we have

$$
\angle \mathrm{C}^{\prime} \mathrm{AB}+\angle \mathrm{B}+\angle \mathrm{C}^{\prime}-\pi=\int_{\partial \triangle \mathrm{ABC} C^{\prime}} \hat{\kappa}_{g} d \tau+\int_{\triangle \mathrm{ABC} C^{\prime}} K d A+2 \int_{\Sigma \cap(\triangle \mathrm{ABC})^{\prime}{ }^{\circ}} \kappa_{s} d \tau,
$$

and

$$
\angle \mathrm{C}^{\prime} \mathrm{AC}+\angle \mathrm{ACC}+\angle \mathrm{C}^{\prime}-\pi=\int_{\partial \triangle \mathrm{ACC} C^{\prime}} \hat{\kappa}_{g} d \tau+\int_{\triangle \mathrm{ACC}^{\prime}} K d A+2 \int_{\Sigma \cap(\triangle \mathrm{ACC})^{\circ}} \kappa_{s} d \tau
$$

Subtracting (3.5) from (3.4), we obtain

$$
\angle \mathrm{A}+\angle \mathrm{B}+\angle \mathrm{C}-\pi=\int_{\partial \triangle \mathrm{ABC}} \hat{\kappa}_{g} d \tau+\int_{\triangle \mathrm{ABC}} K d A
$$


since

$$
\int_{\Sigma \cap\left(\triangle \mathrm{ABC}^{\prime}\right)^{\circ}} \kappa_{s} d \tau-\int_{\Sigma \cap(\triangle \mathrm{ACC})^{\circ}} \kappa_{S} d \tau=\int_{\widehat{\mathrm{AC}}} \kappa_{S} d \tau
$$

and $\angle A C C^{\prime}+\angle \mathrm{C}=\pi$.

This proves the formula (3.2) for any admissible triangle.

\section{The proof of Theorem B}

Proof of Theorem B. Although $\partial M_{+}$and $\partial M_{-}$are the same set, their orientations are opposite. However, the singular curvature $\kappa_{s}$ does not depend on the orientation of the singular curve. So we have

$$
\int_{\partial M_{+}} \kappa_{S} d \tau+\int_{\partial M_{-}} \kappa_{S} d \tau=2 \int_{\Sigma} \kappa_{S} d \tau
$$

The singular set on a sufficiently small neighborhood of a peak $p$ consists of finitely many regular $C^{1}$-curves starting from $p$. (The number $2 m(p)$ of these singular curves starting from $p$ is always even.) Since the integrations of geometric curvatures not on singular curves are cancelled by opposite integrations, we have from Theorem 3.3 that

$$
\begin{aligned}
& 2 \pi \chi\left(M_{+}\right)=\int_{M_{+}} K d A+\int_{\partial M_{+}} \kappa_{s} d \tau+\sum_{p: \text { peak }}\left(m(p) \pi-\alpha_{+}(p)\right), \\
& 2 \pi \chi\left(M_{-}\right)=\int_{M_{-}} K d A+\int_{\partial M_{-}} \kappa_{s} d \tau+\sum_{p: \text { peak }}\left(m(p) \pi-\alpha_{-}(p)\right) .
\end{aligned}
$$

Hence by (4.1), (2.5) and Definition 2.9, we have

$$
\begin{aligned}
& 2 \pi\left(\chi\left(M_{+}\right)+\chi\left(M_{-}\right)\right)=\int_{M^{2}} K d A+2 \int_{\Sigma} \kappa_{s} d \tau+2 \pi \sum_{p: \text { peak }}(m(p)-1), \\
& 2 \pi\left(\chi\left(M_{+}\right)-\chi\left(M_{-}\right)\right)=\int_{M^{2}} K d \hat{A}-\sum_{p: \text { peak }}\left(\alpha_{+}(p)-\alpha_{-}(p)\right) .
\end{aligned}
$$

Since $M^{2}$ is the disjoint union of $M_{+}, M_{-}$and $\Sigma$, the following formula for Euler numbers holds:

$$
\chi\left(M^{2}\right)=\chi\left(M_{+}\right)+\chi\left(M_{-}\right)+\chi(\Sigma) .
$$

Since we assumed that $\Sigma$ admits at most peaks, $\Sigma$ is a finite topological graph. Hence, we have $\chi(\Sigma)=\sum_{p \text { :peak }}(1-m(p))$. Thus, we have

$$
\begin{gathered}
2 \pi\left(\chi\left(M_{+}\right)+\chi\left(M_{-}\right)+\chi(\Sigma)\right)=\int_{M^{2}} K d A+2 \int_{\Sigma} \kappa_{s} d \tau, \\
\chi\left(M_{+}\right)-\chi\left(M_{-}\right)=\frac{1}{2 \pi} \int_{M^{2}} K d \hat{A}-\left(\# P_{+}-\# P_{-}\right),
\end{gathered}
$$

where we used (2.6). Thus, we have (2.9). Finally, by (1.8) and (1.9), we have (2.8). 


\section{REFERENCES}

[CI] S. Chino and S. Izumiya. Lightlike developables in Minkowski 3-space. Preprint.

[FSUY] S. Fujimori, K. Saji, M. Umehara and K. Yamada. Singularities of maximal surfaces, to appear in Math. Z. DOI: 10.1007/s00209-007-0250-0.

[I] G. Ishikawa. Developable of a curve and determinacy relative to osculation-type. Quart. J. Math. Oxford 46 (1995), 437-451.

[IST] S. Izumiya, K. Saji and M. Takahashi. Horospherical flat surfaces in hyperbolic 3-space. Preprint.

[KRSUY] M. Kokubu, W. Rossman, K. Saji, M. Umehara and K. Yamada. Singularities of flat fronts in hyperbolic 3-space. Pacific J. of Math. 221 (2005), 303-351.

[K] M. Kossowski. The Boy-Gauss-Bonnet theorems for $C^{\infty}$-singular surfaces with limiting tangent bundle. Ann. Global Anal. Geom. 21 (2002), 19-29.

[LLR] R. Langevin, G. Levitt and H. Rosenberg. Classes d'homotopie de surfaces avec rebroussements et queues d'aronde dans $\mathbb{R}^{3}$. Canad. J. Math. 47 (1995), 544-572.

[SUY] K. Saji, M. Umehara and K. Yamada. The geometry of fronts. Preprint (2005) math.DG/0503236, to appear in Ann. of Math.

[S] O. P. Scherbak. Wavefront and reflection groups. Russian Math. Surveys 43(3) (1988), 149-194.

Kentaro Saji
Department of Mathematics

Hokkaido University

Sapporo 060-0810, Japan

(E-mail: saji@math.sci.hokudai.ac.jp)

Masaaki Umehara

Department of Mathematics

Graduate School of Science

Osaka University, Toyonaka

Osaka 560-0043, Japan

(E-mail: umehara@math.sci.osaka-u.ac.jp)

\author{
Kotaro Yamada \\ Faculty of Mathematics \\ Kyushu University
}

Higashi-Ku, Fukuoka 812-8581, Japan

(E-mail:kotaro@math.kyushu-u.ac.jp) 